\title{
THE ALMOST FIXED POINT PROPERTY FOR NONEXPANSIVE MAPPINGS ${ }^{1}$
}

\author{
SIMEON REICH
}

\begin{abstract}
It is shown that a closed convex subset of a reflexive Banach space has the almost fixed point property for nonexpansive mappings if and only if it is linearly bounded.
\end{abstract}

Let $C$ be a closed convex subset of a Banach space $(E,|\cdot|)$. Recall that a mapping $T: C \rightarrow E$ is said to be nonexpansive if $|T x-T y| \leqslant|x-y|$ for all $x$ and $y$ in $C$. The set $C$ is said to have the almost fixed point property for nonexpansive mappings if $\inf \{|x-T x|: x \in C\}=0$ for all nonexpansive $T: C \rightarrow C$. Any bounded $C$ has this property (see, for example, [12, p. 35]). The set $C$ is called linearly bounded if it has a bounded intersection with all lines in $E$. The purpose of this note is to characterize those closed convex subsets of reflexive Banach spaces $E$ which possess the almost fixed point property for nonexpansive mappings. The best result to date has been that of Baillon and Ray [2] who assumed that $E$ belongs to a special class of superreflexive spaces. For previous results in this direction see [3] (where $E=l^{2}$ ) and [6] (where $E=l^{p}, 1<p<\infty$ ). Our approach to this problem differs from those used previously. For other aspects of almost fixed point theory see [13].

THEOREM. A closed convex subset of a reflexive Banach space has the almost fixed point property for nonexpansive mappings if and only if it is linearly bounded.

Proof. Let $C$ be a closed convex subset of a (real) reflexive Banach space $E$, and let $E^{*}$ be the dual of $E$. To show necessity, assume that $\{y+t a: 0 \leqslant t<\infty\} \subset C$ for some $a \neq 0$. If $x$ is in $C$, then $(1-1 / t) x+(y+t a) / t$ belongs to $C$ for all $t \geqslant 1$. Therefore we can define a mapping $S: C \rightarrow C$ by $S x=x+a$. This mapping is nonexpansive and $|x-S x|=|a|$ for all $x \in C$.

Conversely, let $T: C \rightarrow C$ be any nonexpansive mapping, and denote $\inf \{|x-T x|: x \in C\}$ by $d$. It is known [11, §4] (see also [5]) that for each $x \in C$ there is a functional $j \in E^{*}$ with $|j|=d$ such that $\left(\left(x-T^{n} x\right) / n, j\right) \geqslant d^{2}$ for all $n \geqslant 1$. (Note that by Banach's fixed point theorem, the accretive operator $I-T$ does indeed satisfy the range condition.) It is also known [10, Proposition 4.3] that $\lim _{n \rightarrow \infty}\left|T^{n} x\right| / n=d$. Let a subsequence of $\left\{T^{n} x / n\right\}$ converge weakly to $w$. Clearly

Received by the editors May 18, 1982. Presented to the Society by title (see Abstracts Amer. Math. Soc. 3 (1982), 402).

1980 Mathematics Subject Classification. Primary 47H09.

$\mathrm{Kev}$ words and phrases. Almost fixed point property, linearly bounded, nonexpansive mapping.

'Partially supported by the National Science Foundation under Grant MCS 81-02086. 
$|w| \leqslant d$. On the other hand, $|w| d=|w||j| \geqslant(-w, j) \geqslant d^{2}$, so that $|w|=d$. Now let $y$ be any point in $C$. Since $(1-1 / n) y+T^{n} x / n$ belongs to $C$ for each $n \geqslant 1$, we see that $y+w$ also belongs to $C$. Consequently, we may conclude that the points $y+m w$ belong to $C$ for all $m \geqslant 1$. If $C$ is linearly bounded, then this fact implies that $w=0$, so that $d=0$ too. This completes the proof.

REMARK 1. The Theorem cannot be extended to all Banach spaces. To see this, let $E=l^{1}, C=\left\{x=\left(x_{1}, x_{2}, \ldots\right) \in l^{1}:\left|x_{n}\right| \leqslant 1\right.$ for all $\left.n\right\}$, and define $T: C \rightarrow C$ by $T\left(x_{1}, x_{2}, \ldots\right)=\left(1, x_{1}, x_{2}, \ldots\right)$. Then $C$ is linearly bounded and $T$ is an isometry, but $\inf \{|x-T x|: x \in C\}=1$.

REMARK 2. For $x \in C$, let $I_{C}(x)=\{z \in E: z=x+a(y-x)$ for some $y \in C$ and $a \geqslant 0\}$, and recall that a mapping $f: C \rightarrow E$ is said to be weakly inward if $f(x)$ belongs to the closure of $I_{C}(x)$ for each $x$ in $C$. We note that if $C$ is a linearly bounded, closed convex subset of a reflexive Banach space $E$, and a nonexpansive $T$ : $C \rightarrow E$ is weakly inward, then $\inf \{|x-T x|: x \in C\}=0$ too. This is true because the proof of [8, Theorem 3.1] shows that the Theorem can be applied to the resolvent $(I+r(I-T))^{-1}: C \rightarrow C$, where $I$ denotes the identity operator and $r$ is positive. Alternatively, we could have established the Theorem and Remark 2 simultaneously by using the results of $\S 2$ of [11]. More generally, the same result is valid for any continuous, weakly inward $T: C \rightarrow E$ such that $I-T$ is accretive.

REMARK 3. A closed convex subset $C$ of a Banach space $E$ is said to have the fixed point property for nonexpansive mappings if every nonexpansive $T: C \rightarrow C$ has a fixed point. We remark in passing that if $E$ is a Hilbert space and $C$ is unbounded, then $C$ does not have this property [7]. If $E$ is either uniformly convex or uniformly smooth, then every bounded closed convex subset of $E$ has the fixed point property for nonexpansive mappings, but it is not known if this is true for all reflexive spaces. For more information concerning this property see [4 and 9]. Note, in particular, that the question discussed in [9] has been recently answered in the negative by Alspach [1].

AcKnowledgement. I am grateful to Professor K. Goebel for several helpful conversations.

NOTE ADDED IN PROOF.

REMARK 4. If $E$ is finite-dimensional and $C$ is linearly bounded, then $C$ is, in fact, bounded. Hence in this case either $C$ is bounded and has the fixed point property, or it is unbounded and does not even have the almost fixed point property for nonexpansive mappings.

REMARK 5. It may be of interest to compare the Banach space situation with that of the Hilbert ball $B$ equipped with the hyperbolic metric $\rho$. In the hyperbolic case, a $\rho$-closed $\rho$-convex subset $K$ of $B$ has the fixed point property (and the almost fixed point property) for $\rho$-nonexpansive mappings if and only if it is geodesically bounded. Hence there are $\rho$-unbounded sets $K$ which have the fixed point property for $\rho$-nonexpansive mappings.

\section{REFERENCES}

1. D. E. Alspach, A fixed point free nonexpansive map, Proc. Amer. Math. Soc. 82 (1981), 423-424.

2. J.-B. Baillon and W. O. Ray, Fixed points and approximate fixed points of nonexpansive mappings, preprint. 
3. K. Goebel and T. Kuczumow, $A$ contribution to the theory of nonexpansive mappings, Bull. Calcutta Math. Soc. 70 (1978), 355-35\%.

4. W. A. Kirk, Fixed point theory for nonexpansive mappings, Fixed Point Theory, Lecture Notes in Math., vol. 886, Springer-Verlag, Berlin and New York, 1981, pp. 484-505.

5. E. Kohlberg and A. Neyman, Asymptotic behavior of nonexpansive mappings in normed linear spaces, Israel J. Math. 38 (1981), 269-275.

6. W. O. Ray, Nonexpansive mappings on unbounded convex domains, Bull. Acad. Polon. Sci. 26 (1978), $241-245$.

7. The fixed point property and unbounded sets in Hilbert space, Trans. Amer. Math. Soc. 258 (1980), 531-537.

8. S. Reich, On fixed point theorems obtained from existence theorems for differential equations, J. Math. Anal. Appl. 54 (1976), 26-36.

9. 266-268; 87 (1980), 292-294.

10. On the asymptotic behavior of nonlinear semigroups and the range of accretive operators, $\mathrm{J}$. Math. Anal. Appl. 79 (1981), 113-126.

11. On the asymptotic behavior of nonlinear semigroups and the range of accretive operators. II, Mathematics Research Center Report \#2198, 1981: J. Math. Anal. Appl. 87 (1982), 134-146.

12. D. R. Smart, Fixed point theorems, Cambridge Univ. Press, Cambridge, 1974.

13. T. van der Walt, Fixed and almost fixed points, Mathematisch Centrum, Amsterdam, 1963.

Department of Mathematics, University of Southern California, los angeles, Californi. 90007 